\title{
Robo-Tipping: Are Customers Game?
}

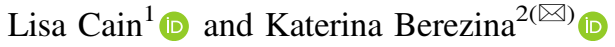 \\ ${ }^{1}$ Florida International University, Miami, FL 33181, USA \\ lcain@fiu.edu \\ ${ }^{2}$ University of Mississippi, University, MS 38677, USA \\ katerina@katerinaberezina.com
}

\begin{abstract}
This study sought to investigate customer attitudes towards tipping robotic employees in bars. A convenience sample of participants who were 21 years of age or older and who had patronized a bar was recruited using Amazon's Mechanical Turk platform. Of the 102 usable responses, only 15 participants had experienced robotic bartender service. Only 11 individuals $(10.8 \%)$ in total said they would tip a robot; 10 of those were respondents who had acutally experienced robotic bartenders, representing $67 \%$ of that subsample. Participants listed efficiency and required maintenance as reasons for giving a tip to a robotic bartender. Out of 91 respondents who initially declined to tip a robotic bartender, 38 study participants $(41.8 \%)$ agreed to tip if they knew that the collected funds would go to human employees. However, in the same group of respondents, only $14(15.4 \%)$ agreed to tip in the scenario when tipping would serve as a learning experience for a robot to understand customer preferences. The rationale for not tipping included such reasoning as robots are machines that do not need extra income and cannot appreciate the gesture. This study suggests that explaining how the collected funds will be used may positively impact consumer intentions to tip a robotic bartender. This extra revenue may help offset the cost of the robot, and subsequently lower the prices of the drinks served at a robotic bar, thus making the product more affordable for a wider audience.
\end{abstract}

Keywords: Robotic employees - Robotic bartender - Tipping behavior

\section{Introduction}

Robotics and automation have entered the hospitality industry, which has been traditionally regarded as a people industry $[6,8,9]$. Currently, robots are employed as hosts, wait staff, food runners, cooks, and bartenders [5]. However, customer acceptance of and reaction to this new type of employee in bar settings has not been studied. Because the beverage service industry in the United States relies on a tipping culture, understanding how employing robots impacts customers' service evaluations and tipping practices is of importance. Therefore, the purpose of this study was to investigate customer attitudes towards tipping robotic employees in bars. 


\section{Literature Review}

Tipping is a complex phenomenon that involves the organization, employees, and customers. From the perspective of the agency theory, tipping may serve as a mechanism that, which assists in aligning the interests of the service company (the principal) and the employee (the agent) [2]. While the principal is usually interested in business success that may be defined via financial performance, customer satisfaction, customer return, or business reputation, agents are usually more concerned about their personal benefits that may be expressed via working hours, work conditions, and high pay. In such a goal conflict, tips appear to be an instrument that rewards employees with higher income for providing high quality service to customers, which, in turn, may satisfy the business owner expectations of increased earning, customer satisfaction, and return.

The logic presented above seems to work well when all parties in the organization employee - customer relationship are human. However, the introduction of robotic employees ensures a perfect goal alignment between the principal and the agent, and removes any personal interests of the agent. In such a scenario, the robotic employee is not interested in receiving tips because it is a machine and does not have personal interests. However, the organization may remain interested in receiving tips as an additional source of revenue that recognizes the quality of product and service that customers received. The funds received as tips may be used by the organization in a variety of ways, such as offsetting the cost of robotic employees or supporting human employees who remain in the organization. Additionally, if tips are going directly to the organization, the company may choose to lower its prices accordingly to attract more customers and increase value perception. Therefore, the question is whether or not customers would be willing to tip robotic employees.

Previous literature has established that tipping is a complex multi-faceted behavior [4]. Even though numerous studies have examined the tipping behavior of patrons (e.g., see 2, 14 for meta-analysis), no comprehensive theoretical framework has been developed in this area of human behavior [4]. The studies conducted in this area usually approach the topic from either psychological or economic perspectives.

Extant literature on the psychological motivations behind tipping includes the desire to reward good service, assist servers financially, and increase social status or gain approval [11, 12, 15]. While scholars have examined moderators of the servicetipping relationship including, but not limited to, gender and ethnicity of the server, time of service, age of the patron, and bill size [13]. Tipping scholars have also sought to identify how best to increase tips for service staff [10]. Ultimately, research has demonstrated that behavioral changes, such as self-introductions, eye contact, offering chocolates, and drawing suns on the receipts may lead to positive changes in customer tipping behavior (increased tip percentage) [4, 7, 17].

The economic perspective usually attempts to test the assumption that tipping is a rational behavior that rewards high quality service [4]. However, meta-analytic studies reveal a significant but weak relationship between service quality and the tip percentage $[2,14]$, therefore, suggesting otherwise. Furthermore, Azar [2] was not able to find support for the relationship between repeat visits to an establishment and tipping behavior as a mechanism of ensuring better service in the future. Becker et al. [4] 
conducted regression modeling to identify factors that predict customer tipping behavior, and identified that the heuristics model was the factor with the highest regression coefficients making statistically significant contributions to models built for exceptional, satisfactory, and poor service.

In summary, customer tipping behavior appears to be a complex, possibly irrational behavior that is often driven by heuristics and may be influenced by psychological manipulations. Based on the findings documented in the previous literature, one may suggest that customers may choose to tip robotic employees for some pre-existing innate reasons (e.g., personal beliefs, cultural environment, etc.). Therefore, this study seeks to explore customer baseline attitudes toward giving tips to robotic employees, and customer susceptibility to being influenced by psychological factors.

\section{Methods}

To achieve the purpose of this study, an online survey was built on Qualtrics. The survey included a combination of multiple-choice and open-ended questions. In the survey, participants were asked to watch a video about a robotic bartender and answer questions about their perceptions of the robotic bartender and whether or not they would tip, and why. Additionally, the study participants were asked if they would tip a robotic bartender if they knew that the collected funds would go to human employees or serve as a learning experience for the robot to understand customer likes and dislikes.

A convenience sample of participants who were located in the United States, 21 years of age or older and who had patronized a bar was recruited using Amazon's Mechanical Turk (MTurk) platform. To increase the quality of responses, participants were also required to have an approval rate of previous requests of $95 \%$ or above [16]. In addition to that, the survey contained a combination of multiple-choice and openended questions. The study design made it easy to eliminate low quality responses through the review of qualitative answers.

\section{Results}

A total of 122 responses were collected for this study in October, 2019. Eighteen responses were incomplete, and therefore were excluded from the study. Two more respondents provided ineligible qualitative answers (a string of characters) and were subsequently eliminated from the analysis as well. Of the 102 usable responses, 15 respondents $(14.7 \%$ ) had experienced a robotic bartender at one of the casino or cruise line bars at which they are featured. Of those, 10 (67\%) said that they would tip a robot. Reasons mentioned included, "Yes: It hardly spills and it's quick and easy; its humanity; Yes, that tip is not for the robot, it's for bar and robot maintenance." Only 11 individuals $(10.8 \%)$ in total said they would tip. The one individual who had not experienced a robotic bartender in person before, but said they would tip offered the rationale: "to see if there would be a positive reaction to my tip." 
The remaining 91 individuals who said they would not tip a robot overwhelmingly provided responses like "Robots don't need income to survive", "I would only want to tip a human", and "When I tip, it's because a person who's serving me isn't making minimum wage and tips are there to compensate of that." However, some respondents cited a lack of gratitude as a reason for not tipping: "the robot is not human so it would not appreciate a tip or do anything with it." Another participant cited, "it doesn't have a soul."

Out of 91 respondents who initially declined to tip a robotic bartender, 38 study participants $(41.8 \%)$ agreed to tip if they knew that the collected funds would go to human employees. However, in the same group of respondents, only $14(15.4 \%)$ agreed to tip in the scenario when tipping would serve as a learning experience for a robot to understand customer likes and dislikes.

\section{Discussion, Implications, and Conclusion}

\subsection{Theoretical Contribution}

This exploratory study was designed to measure customers' intentions to tip robotic emplyees and explore possibilities of influencing such intentions. The results of the study revealed that $10.8 \%$ of respondents were willing to tip robotic bartenders. Interestingly, the willingness to tip seems to be higher among those customers who have experienced a service offered by a robotic bartender (67\%). Therefore, it appears that experience with a robotic employee makes a difference in a customer's intention to offer tips.

Also, an intention to tip a robotic bartender increased after offering explanations that tips would serve as a learning experience for the robot to understand customer preferences $(15.4 \%)$ or support human employees $(41.8 \%)$. The findings of the study seem to be in line with the results of previous research on tipping human emplyees that could not establish a link between tipping behavior and future visits to the establishment [2]. The first scenario suggests that a robot may learn from the tips given to it which service situations were evaluated positively by customer and which ones were not up to the par, thus, resulting in future improvements of service. This scenario generated a small increase in customer willingness to tip a robotic bartender, therefore, suggesting that improvements of future service encounters is not a strong motivator for offering tips to robots.

The second scenario that suggested that collected tips would be used to support human employees generated a higher increase in the intention to tip a robotic bartender. First, such a scenario may be more familiar to the participants. Different businesses may use different tip distribution strategies, however, tips going to human employees has been a status quo in the industry. Second, this scenario may speak to the customers' gratitude, empathy, and desire to help service employees that has been documented in the literature as motives for tipping $[1,11,12,15]$. Overall, these two scenarios suggest that customer intentions to tip robotic employees may be altered by different psychological influences, which has also been documented for tipping human employees $[4,7,17]$. 


\subsection{Practical Implications}

From the practical perspective, because respondents cited efficiency and maintenance as reasons for tipping, practitioners need to ensure the machinery runs seamlessly and is constantly updated to drive customer satisfaction. It is also important to ensure that gratitude or some other reaction is expressed on the part of the machine as "a reaction" was cited as a reason for offering a tip. The rationale provided for not tipping robots supports discussions put forth by Lynn and McCall [14] who maintain that gratitude expressed by the server is a necessary component.

The findings of this study also suggest that bar operators may expect to receive extra revenue in tips from around $11 \%$ of customers. This percentage may increase in the category of repeat customers or those who have experienced a service by a robotic employee in the past. This extra revenue may help to offset the cost of the robot, and, therefore, lower the prices of the drinks served at a robotic bar, thus making the product more affordable for a wider audience. Additionally, this study suggests that explaining the way how the collected funds will be used, may positively impact consumer intentions to leave a tip for a robotic bartender.

\subsection{Limitations and Future Research}

This study was limited by a small sample size and convenience sampling method, which both limit generalizability. Future research should attempt to gather information from more individuals who have and have not encountered robotic service to assess how this impacts customers overall service evaluation and tipping of employees who work alongside robots. Identifying how these robotic interactions are perceived and valued by customers is important for both internal and external customer service.

The tipping literature has been criticized for the lack of sound theoretical frameworks that could explain tipping behavior [3,4]. Such a void is even larger for the area of tipping robotic employees and understanding customer behavior in new, emerging service environments. Therefore, future research should concentrate on developing theoretical frameworks that could offer insight into customers' intentions of tipping robotic employees. Such studies may rely on the extant tipping literature and approach the question from either psychological or economics perspectives. Additionally, future research may leverage the literature on human-robot interactions and consider such aspects as anthropomorphic features of robots or speech abilities.

\section{References}

1. Azar $\mathrm{OH}$ (2005) Who do we tip and why? An empirical investigation. Appl Econ 37 (16):1871-1879

2. Azar OH (2008) Strategic behavior and social norms in tipped service industries. BE J Econ Anal Policy $8(1): 1-16$

3. Banks GC, Woznyj HM, Kepes S, Batchelor JH, McDaniel MA (2018) A meta-analytic review of tipping compensation practices: an agency theory perspective. Pers Psychol 71 (3):457-478 
4. Becker C, Bradley GT, Zantow K (2012) The underlying dimensions of tipping behavior: an exploration, confirmation, and predictive model. Int J Hosp Manag 31(1):247-256

5. Berezina K, Ciftci O, Cobanoglu C (2019) Robots, artificial intelligence and service automation in restaurants. In: Ivanov S, Webster C (eds) Robots, artificial intelligence and service automation in travel, tourism and hospitality. Emerald Publishing, London, pp 185219

6. Cain LN, Thomas JH, Alonso M Jr (2019) From sci-fi to sci-fact: the state of robotics and AI in the hospitality industry. J Hosp Tour Technol 10(4):624-650

7. Gueguen N, Legoherel P (2000) Effect on tipping of barman drawing a sun on the bottom of customers' checks. Psychol Rep 87(1):223-226

8. Ivanov SH, Gretzel U, Berezina K, Sigala M, Webster C (2019) Progress on robotics in hospitality and tourism: a review of the literature. J Hosp Tour Technol 10(4):489-521

9. Ivanov SH, Webster C, Berezina K (2005) Adoption of robots and service automation by tourism and hospitality companies. Revista Turismo Desenvolvimento 27(28):1501-1517

10. Lynn M (2005) Increasing servers' tips: what managers can do and why they should do it. J Foodserv Bus Res 8:89-98

11. Lynn M (2006) Tipping in restaurants and around the globe: an interdisciplinary review. In: Altman M (ed) Handbook of contemporary behavioral economics: foundations and developments. M.E. Sharpe Publishers, Armonk, pp 626-643

12. Lynn M (2009) Individual differences in self-attributed motives for tipping: antecedents, consequences, and implications. Int J Hosp Manag 28(3):432-438

13. Lynn M, Jabbour P, Kim WG (2012) Who uses tips as a reward for service and when? An examination of potential moderators of the service-tipping relationship. J Econ Psychol 33 (1):90-103

14. Lynn M, McCall M (2000) Gratitude and gratuity: a meta-analysis of research on the service-tipping relationship. J Socio-Econ 29:203-214

15. Saunders SG, Lynn M (2010) Why tip? An empirical test of motivations for tipping car guards. J Econ Psychol 31(1):106-113

16. Stone AA, Walentynowicz M, Schneider S, Junghaenel DU, Wen CK (2019) MTurk participants have substantially lower evaluative subjective well-being than other survey participants. Comput Hum Behav 94:1-8

17. Strohmetz DB, Rind B, Fisher R, Lynn M (2002) Sweetening the till: the use of candy to increase restaurant tipping 1. J Appl Soc Psychol 32(2):300-309

Open Access This chapter is licensed under the terms of the Creative Commons Attribution 4.0 International License (http://creativecommons.org/licenses/by/4.0/), which permits use, sharing, adaptation, distribution and reproduction in any medium or format, as long as you give appropriate credit to the original author(s) and the source, provide a link to the Creative Commons license and indicate if changes were made.

The images or other third party material in this chapter are included in the chapter's Creative Commons license, unless indicated otherwise in a credit line to the material. If material is not included in the chapter's Creative Commons license and your intended use is not permitted by statutory regulation or exceeds the permitted use, you will need to obtain permission directly from the copyright holder.

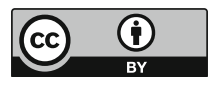

